\title{
Strates
}

STRATES Matériaux pour la recherche en sciences sociales

$8 \mid 1995$

La question de l'environnement: recherches parallèles en Espagne et en France

\section{Politiques de protection des espaces naturels}

Le cas andalou

Francisco José Díaz Ojeda et Juan Francisco Ojeda Rivera

\section{(2) OpenEdition}

Journals

Édition électronique

URL : http://journals.openedition.org/strates/978

DOI : $10.4000 /$ strates. 978

ISSN : $1777-5442$

Éditeur

Laboratoire Ladyss

Édition imprimée

Date de publication : 30 juin 1995

ISSN : 0768-8067

Référence électronique

Francisco José Díaz Ojeda et Juan Francisco Ojeda Rivera, «Politiques de protection des espaces naturels », Strates [En ligne], 8 | 1995, mis en ligne le 20 décembre 2005, consulté le 08 septembre 2020. URL : http://journals.openedition.org/strates/978 ; DOI : https://doi.org/10.4000/strates. 978

Ce document a été généré automatiquement le 8 septembre 2020

Tous droits réservés 


\section{Politiques de protection des espaces naturels}

Le cas andalou

Francisco José Díaz Ojeda et Juan Francisco Ojeda Rivera

\section{NOTE DE L'ÉDITEUR}

Traduit de l'espagnol par Marie-Laure Drouet et Michèle Petit et relu par Aline Brochot.

1 Dans leur conception originelle, propre aux pays neufs caractérisés par de grandes disponibilités en terres et un faible peuplement, les «espaces protégés " sont des «réserves» de nature vierge. Le transfert du concept en Europe l'a fait évoluer vers une acception plus dynamique, d'où l'homme n'est pas exclu. Dès lors, toute action de conservation ne peut se faire sans prendre en compte l'ensemble des interactions entre l'homme et l'espace protégé.

2 Cette évolution conceptuelle s'est traduite, au niveau de l'Etat espagnol et des Régions autonomes, par la mise en place d'un cadre législatif qui définit les objectifs généraux et les modalités d'intervention publique de la politique d'environnement. Trois types de politiques sont distinguées :

* La politique de protection pure ou sanctuarisation: elle concerne des espaces peu modifiés par l'homme, où les activités humaines sont strictement limitées, et où l'objectif est de maintenir l'intégrité d'écosystèmes de grande fragilité. Dans ce type d'espaces économiquement peu exploités, cette protection stricte ne génère pas de conflit avec la population locale du fait de restrictions d'usage.

$4{ }^{*}$ La politique de compensation sociale: lorsque l'objectif de protection porte sur des territoires plus anthropisés, des problèmes spécifiques peuvent apparaitre, du fait de l'existence d'activités économiques dont l'intérêt, actuel et futur, peut aller à l'encontre des objectifs de conservation. Les conflits avec la population rurale résidente 
portent alors sur les restrictions d'usage imposées par la protection de l'espace, et le manque à gagner qui en découle.

Dans un premier temps, les pouvoirs publics prennent acte de la dichotomie entre conservation et développement, sans proposer d'alternative aux modèles traditionnels. La réduction des conflits entre les intérêts de la population qui exploite ces ressources et l'intérêt général s'effectue par le biais de politiques d'investissements bénéficiant directement à la population concernée, en compensation du dommage économique causé.

$6{ }^{*}$ La politique d'« intégration» des intérêts locaux et de l'intérêt général: il est possible d'apporter une réponse qui intègre les intérêts en jeu lorsque l'on peut s'appuyer sur des modèles alternatifs de développement économique, ou du moins tenter d'en élaborer. La construction de ces modèles part d'une conception nouvelle de l'espace naturel, qui est considéré comme le produit de l'action combinée des conditions naturelles et de la transformation anthropique. Une telle approche conduit à analyser et à valoriser les systèmes traditionnels de production et d'organisation sociale qui ont permis le maintien des ressources naturelles, à un niveau d'équilibre tel qu'aujourd'hui ces territoires sont considérés comme dignes d'être protégés.

7 Ce retour sur le passé, à la recherche des principes de base de l'organisation de ces espaces, doit soutenir l'actuelle politique d'intégration des différents objectifs, pour établir les fondements théoriques d'instruments de mise en valeur qui rendent compatibles la protection et le développement économique. Cette politique repose par ailleurs sur le constat qu'il est illusoire de vouloir mener une action de protection ambitieuse sans l'adhésion de la population concernée.

8 Tenter de recomposer les structures sociales et culturelles et d'infléchir les modes d'organisation, telle doit être la ligne prioritaire et indispensable de l'intervention publique, afin de permettre l'introduction et la mise au point de mesures susceptibles d'avoir un impact direct sur la productivité du système économique de ces régions même si cet objectif n'est envisageable qu'à long terme.

9 Mettre à profit le potentiel propre aux espaces protégés et prendre en considération les nouvelles tendances du marché où « le vert fait vendre » devient une opportunité réelle pour les petites entreprises capables de s'adapter à une demande toujours plus exigeante quant à la qualité des produits. La diversification et la personnalisation d'une offre de qualité de produits et de services "naturels" spécifiques à ces espaces (tourisme rural, industries agro-alimentaires de transformation, élaboration de produits artisanaux...) constituent dès lors pour les pouvoirs publics des facteurs de dynamisation des structures socio-économiques des communes touchées par la politique de protection.

Le cadre légal : caractère généraux de la loi nationale $4 / 89$ et les nouveautés introduites par la loi andalouse $2 / 89$

10 En matière d'environnement, le cadre juridique national est la loi 4/89 du 27 mars, relative à la Conservation des espaces naturels protégés et de la flore et de la faune sauvages, rédigée en complément des principes et exigences de l'article 45 de la Constitution espagnole qui stipule :

11 1. «Toute personne a le droit de jouir d'un environnement adéquat à son propre développement, ainsi que le devoir de le conserver. 
12 2. Les pouvoirs publics veilleront à l'utilisation rationnelle de toutes les ressources naturelles, afin de protéger et améliorer la qualité de la vie et de préserver et restaurer l'environnement en s'appuyant sur une indispensable solidarité publique.

13 3. En cas de violation des dispositions antérieures, des sanctions pénales ou administratives, ainsi que l'obligation de réparer les dommages causés, seront édictées. "

14 Dans cette perspective, la loi 4/89 crée un cadre juridique visant à protéger les ressources naturelles, sans toutefois en restreindre, le plus souvent, l'exploitation, dans le but d'un développement économique et social raisonné.

15 Parmi les particularités essentielles de la loi 4/89, celles qui suivent sont plus largement développées dans le texte :

16 - concevoir la nature non seulement comme le milieu où s'effectuent les processus écologiques, mais aussi comme un ensemble de ressources ;

17 - confier à l'Administration la protection et la conservation de la nature en tant que patrimoine de l'humanité ;

18 - assurer l'aménagement de l'environnement par la mise en place d'un ensemble de régimes de protection qui répondent aux divers aspects de la conservation, et d'une série d'instruments de planification;

19 - l'article 149.1.23. de la Constitution fixe, au niveau national, les compétences en matière d'environnement, à travers un ensemble de régimes de protection et d'instruments de planification. Sur cette base, les Communautés autonomes peuvent déployer les mesures de conservation qui sont statutairement de leur ressort.

20 Mais la nouveauté la plus importante de la loi 4/89 est l'apparition d'un instrument spécifique de planification environnementale: le Plan d'Aménagement des Ressources Naturelles (P.O.R.N. ${ }^{1}$ ), instrument souple qui permet d'intervenir de façon prioritaire et globale, et selon un degré d'intensité variable, dans des espaces déterminés, pour la conservation et la restauration des ressources naturelles.

21 Accompagnant cet instrument, le Plan Directeur d'Usage et de Gestion (P.R.U.G. ${ }^{2}$ ) fixe les orientations de développement, ainsi que les articulations entre les règles d'usage et de gestion, et l'administration de l'espace protégé.

22 En relation directe avec ces dispositions, une classification des espaces naturels protégés, bien que très vague, est introduite. Elle s'opère en fonction de la superficie, de la nature et de la valeur des biens à protéger :

23 * Parcs naturels : ce sont des régions naturelles peu modifiées par l'exploitation ou l'occupation humaine qui, en raison de la beauté de leurs paysages, de la représentativité de leurs écosystèmes, ou de la spécificité de leur flore, de leur faune ou de leurs formations géomorphologiques, possèdent des valeurs écologiques, esthétiques, pédagogiques et scientifiques, et dont la conservation mérite une attention particulière.

24 * Réserves naturelles: leur création a pour objectif la protection d'écosystèmes, de communautés ou d'éléments biologiques qui, par leur rareté, leur fragilité, leur importance ou leur spécificité, méritent une valorisation particulière.

25 * Monuments naturels: ce sont des espaces ou des éléments naturels d'une notoire singularité, rareté ou beauté, qui justifient une protection spéciale. Sont considérés aussi comme Monuments naturels les formations géologiques, les gisements 
paléontologiques, et les formations géomorphologiques qui présentent un intérêt particulier par leur spécificité ou leur valeur scientifique, culturelle ou paysagère.

* Paysages protégés: il s'agit de sites naturels qui, par leurs qualités esthétiques et culturelles, méritent une protection particulière.

La promulgation de la loi 4/89 du 27 mars modifie, en un certain sens, le cadre politique andalou et, en vertu des compétences établies statutairement en la matière, la Communauté autonome d'Andalousie a adopté, trois mois plus tard, la loi 2/89 du 18 juillet, "qui approuvé l'Inventaire des Espaces Protégés d'Andalousie et qui fixe des mesures additionnelles pour leur protection».

28 Cette loi andalouse développe et complète la législation nationale, confirmant et élargissant, par ailleurs, l'idée de conservation et de développement local : selon celleci, la croissance économique doit s'effectuer de façon que le profit tiré de l'exploitation raisonnée des ressources naturelles revienne aux communes affectées par la décision de protection. A cette fin, l'implication des différents partenaires économiques est nécessaire, comme le stipule l'exposé des motifs de la loi : « la politique de l'administration resterait vide de contenu s'il lui manquait l'appui de la population... "

Pour donner à la conception planificatrice de la loi 4/89 une perspective économique et sociale qui la rende opératoire, la loi 2/89 ajoute aux instruments de planification nationale (P.O.R.N. et P.R.U.G.) deux nouveaux instruments :

30 - le Plan de Développement Intégral ${ }^{3}$, dont la finalité est la dynamisation des structures socio-économiques, tout en sauvegardant la stabilité écologique ;

31 - les Programmes de mise en valeur ${ }^{4}$, qui précisent quelles doivent être la mise en oeuvre, les priorités et les formes particulières des lignes d'actions prévues dans le P.D.I.

32 Par ailleurs, bien que de façon floue, la législation autonome complète la liste des régimes de protection, en lui incorporant les Parcs périurbains, les Réserves naturelles concertées, et les Sites naturels (loi du 2 mai 1975 relative aux Espaces naturels protégés):

33 * Parcs périurbains: ce sont des espaces naturels situés à proximité d'un centre urbain, qu'ils aient été ou non créés ex-nihilo, et dont il convient d'adapter l'utilisation aux besoins des populations en espaces de récréation.

34 * Réserves naturelles concertées: il s'agit d'espaces qui, sans réunir les critères qui caractérisent les formes décrites précédemment, méritent une protection particulière et dont les propriétaires sollicitent l'application d'un régime de protection concertée.

35 * Sites naturels : ce sont des espaces qui, eu égard à leur exceptionnelle valeur, sont classés dans le but de préserver leur flore, leur faune, leur structure géomorphologique, leur beauté spécifique ou d'autres composantes de très haute valeur naturelle.

36 Au total, ce sont $17,2 \%$ du territoire de la Communauté autonome andalouse qui sont classés au titre d'Espaces naturels protégés, soit approximativement 1,5 million d'hectares, constitués, au moment de la promulgation de la loi, de 22 Parcs naturels, 28 Réserves naturelles, 31 Sites Naturels et un Parc national, auxquels se sont progressivement ajoutés des Parcs périurbains, Réserves concertées, ainsi que d'autres espaces actuellement en phase d'étude pour leur classement ultérieur.

Les caractéristiques des Parcs naturels 
37 Parmi les différentes catégories d'espaces naturels protégés, les Parcs naturels peuvent être considérés comme les plus représentatifs, parce qu'il s'agit de territoires vivants, avec une population plus ou moins dense, qui sont de ce fait les espaces où l'Administration fait porter la majeure partie de ses efforts de planification et de gestion. Il est important de le souligner, puisque les dimensions et les particularités des autres types d'espaces naturels protégés - qui sont des espaces restreints - ont donné lieu, jusqu'à aujourd'hui, à des politiques purement conservatrices, ou qui intègrent conservation et gestion pour l'intérêt général, ce qui, a priori, ne requiert pas de grands efforts de planification et de gestion.

Les Parcs naturels présentent les particularités suivantes: leur étendue est vaste, équivalente, dans de nombreux cas, à celle de comarcas ${ }^{5}$; des communautés directement attachées à l'exploitation traditionnelle des ressources naturelles et du territoire y sont établies; la plupart du temps, leur position est excentrée par rapport aux grands centres et aux régions économiquement les plus dynamiques, surtout $\mathrm{du}$ fait des conditions physiques; ce sont, d'une façon générale, des zones socialement et économiquement marginales.

39 En raison de ces particularités, la planification, la gestion et les politiques d'intervention dans ces territoires se distinguent de façon significative de celles qui s'appliquent aux autres espaces naturels protégés. En effet, il est évident que les problèmes dérivés de la conservation ne peuvent être dissociés de ceux qui sont liés à la marginalité sociale et économique, surtout si l'on prend en compte les limites et les entraves au développement de ces territoires :

40 - dépeuplement progressif, abandon des activités traditionnelles, et vieillissement de la population ;

41 - faible productivité des activités primaires traditionnelles, du fait des structures d'exploitation et du manque de diversification des productions - la monoculture est prédominante, avec ce que cela suppose de dépendance aux fluctuations du marché ;

42 - utilisation excessive et anarchique des ressources naturelles, par manque de planification et de rationalisation, et absence d'exploitation raisonnée des ressources cynégétiques ;

43 - manque d'organisation globale et de diversification de la filière agricole et forestière, dans des zones essentiellement vouées à l'exportation de matières premières ;

44 - systèmes d'organisation de type coopératif peu consolidés ;

45 - rigidité excessive dans les décisions relatives à la production ;

46 - déficience des systèmes et circuits de commercialisation des produits locaux ;

47 - quasi-absence, et très faible diversification, des secteurs secondaire et tertiaire ;

48 - déficit important en infrastructures de communication, en équipements urbains (assainissement, approvisionnement, traitement des déchets...) et en services publics ;

49 - quasi-inexistence ou inadéquation des infrastructures pour l'exploitation rationnelle des ressources touristiques ;

50 - faible qualification professionnelle de la population (taux élevé d'analphabétisme). Les instruments de planification relatifs aux Parcs naturels

51 L'ensemble des caractéristiques qui viennent d'être décrites se manifestent, avec plus ou moins d'acuité, dans tous les Parcs naturels. Elles supposent un état de dégradation 
de l'organisation économique et sociale auquel il est difficile de remédier, dès lors qu'il résulte de mécanismes complexes consolidés au fil du temps. La nécessité d'intégrer ces espaces protégés aux structures productives et au marché actuels est fondamentale et déterminante, si l'on veut rendre opératoires les mesures que les pouvoirs publics souhaitent mettre en place.

Tenant compte de ces handicaps et de ces nécessités, la planification relative à l'environnement se déroule à l'aide d'une chaîne d'instruments qui ne prend sa véritable cohérence que dans sa globalité. On ne peut analyser ou mettre en oeuvre l'un de ses maillons sans se référer à ceux qui le précèdent ou le suivent.

Si l'on considère cette planification dans une perspective verticale, en se limitant au régime de protection des Parcs naturels, nous trouvons au sommet les Plans d'Aménagement des Ressources Naturelles, puis les Plans Directeurs d'Usage et de Gestion, et à la base, les Plans de Développement Intégral et les Programmes de mise en valeur.

Plans d'Aménagement des Ressources Naturelles (P.O.R.N.)

L'apparition de cette forme de planification est d'une grande importance, car jusqu'à la promulgation de la loi $4 / 89$, les premiers instruments de planification pour la protection de l'environnement étaient apparus dans le cadre de la planification urbaine. Le champ d'application du P.O.R.N., comparé à celui des autres instruments de planification territoriale et urbaine, lui confère un rôle prédominant, rôle qui est fixé dans la loi 4/89 elle-même, et qui ne fait aucun doute. Ainsi, dans son article 5.2, il est précisé : "Les P.O.R.N. seront obligatoires et exécutifs dans les domaines concernés par la présente loi, ses dispositions s'imposant pour tous les autres instruments de planification territoriale... »

55 La puissance des P.O.R.N. comme instruments normatifs se traduit également dans leur rapport à la propriété privée. Si celle-ci est reconnue, elle se voit limitée par la fonction sociale de l'espace protégé, la législation pouvant alors réglementer l'utilisation de ces biens.

56 En résumé, le P.O.R.N. constitue un cadre normatif dans les aspects liés à l'environnement, et une sorte de schéma directeur pour les autres plans sectoriels ou territoriaux.

Plans Directeurs d'Usage et de Gestion (P.R.U.G.)

57 La loi nationale 4/89, d'autre part, introduit les Plans Directeurs d'Usage et de Gestion, bien qu'elle n'explicite pas leur champ d'application, leurs objectifs, contenus, effets... Du point de vue de la législation des Régions autonomes, l'objectif des P.R.U.G. est de concrétiser à l'échelle adéquate, et avec la précision suffisante, les résolutions générales du P.O.R.N., les articulations entre les règles d'usage et de gestion, et l'administration de l'espace protégé, l'organisation spatiale et la gestion des ressources, en tenant compte des facteurs spécifiques au parc concerné. Il s'agit d'un instrument de gestion directe des ressources naturelles, et d'organisation de l'administration et de la gestion du Parc naturel, qui est en liaison étroite avec l'autorité compétente.

Plans de Développement Intégral (P.D.I.)

Les Plans de Développement Intégral, émanants de la loi autonome 2/89 (Art. 20), se posent comme des instruments dépassant la dichotomie entre conservation et exploitation, qui optent pour un modèle de développement dans lequel les aspects territoriaux, économiques et environnementaux ne sont pas des ensembles distincts 
qui s'opposent, mais les termes inséparables d'un véritable développement à long terme.

La finalité de ces Plans est, précisément, de faire apparaître les possibilités de développement endogène de chaque Parc ainsi que son aire d'influence, en tenant compte de tous les types de ressources disponibles (naturelles, urbaines, humaines...), et de sa position dans le contexte régional, national, technologique, financier. De ce point de vue, les P.D.I. ont pour objet de dynamiser les structures socio-économiques des Parcs naturels, dans le respect des directives et des normes de protection établies au préalable. Les Plans énoncent les alternatives les plus viables pour chaque territoire et communauté, et détaillent les orientations et secteurs prioritaires pour le développement économique. A ce niveau, la Planification Intégrale exige un effort particulier de coordination technique et politique entre les divers départements de l'administration publique d'une part, et entre celle-ci et les partenaires sociaux concernés et impliqués de l'autre.

Programmes de mise en valeur

Les lignes d'action indiquées dans le P.D.I. se concrétisent dans les Programmes de mise en valeur compris comme instruments exécutifs de la planification économique de chaque Parc et de son aire d'influence socio-économique. L'Institut de mise en valeur de l'Andalousie ${ }^{6}$, conformément à l'article 20.3 de la loi $2 / 89$ et à son propre champ de compétences dans le domaine du développement économique, élaborera ainsi ces programmes qui développent les axes des P.D.I. relatifs à la mise en valeur de secteurs économiques déterminés et au renforcement du tissu des entreprises. Il faut noter que l'Institut de mise en valeur de l'Andalousie envisage de créer des Agences de promotion dans chaque Parc, qui seront responsables de l'application du programme prévu.

Sur la base du diagnostic effectué dans les P.D.I., la mise en évidence des principaux problèmes et points de blocage, tant dans le fonctionnement des écosystèmes que dans celui du système socio-économique et territorial permettra, compte tenu des diverses potentialités, de fixer les objectifs généraux et les stratégies de développement qui seront appliquées dans les différents programmes d'action.

Schéma de La politique de planification environnementale

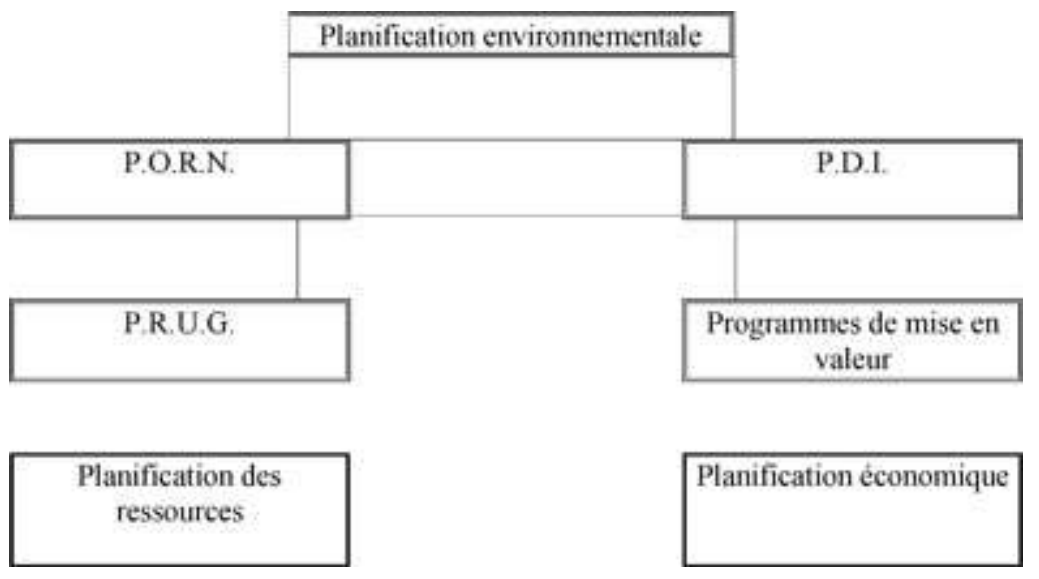

Le schéma général de ce qui a été exposé peut se concrétiser de la façon suivante :

Objectifs relatifs à l'environnement :

- correction des déséquilibres environnementaux ; 


\section{planification, tâche encore plus difficile si l'on prend en compte les particularités du} cas andalou.

77 le sens où il trouve son fondement légal dans l'article 15.2 de la loi 4/89, qui permet de procéder à ce classement sans approbation préalable du P.O.R.N., sous réserve que ce plan soit initié dans un délai d'un an après le classement.

$78{ }^{*}$ La loi 2/89 du 18 juillet, par laquelle l'Inventaire des Espaces naturels protégés est approuvé, concerne un ensemble de 22 Parcs Naturels, parmi lesquels 5 ont été classés antérieurement à cette loi : Sierra de Grazalema (Décret 316/84), Sierra de Cazorla, Segura y Las Villas (Décret 10/86), Sierra de María (Décret 236/87), Cabo de Gata-Nijar (Décret 314/87) et Sierras Subbéticas (Décret 232/88).

79 Deux d'entre eux - Sierra de Grazalema et Sierra de Cazorla, Segura y Las Villas avaient déjà leurs propres instruments de planification (Plans Directeurs d'Usage et de Protection) avant la promulgation de la loi autonome, et le processus actuel n'a donc concerné que 20 des 22 Parc naturels andalous.

$80{ }^{*}$ L'accord du Conseil de gouvernement du 30 janvier 1990, autorisant l'Agence de l'Environnement (A.M.A.') à élaborer les P.O.R.N. des Espaces naturels protégés $\mathrm{d}$ 'Andalousie, indique la procédure à suivre (voir le schéma) :

81 - Les avant-projets des P.O.R.N sont remis pour approbation provisoire au Comité d'Actions Intégrées pour l'Ecodéveloppement (C.A.I.E.), qui a pour fonctions d'impulser, coordonner, suivre et évaluer les programmes visant à harmoniser le développement socio-économique et les exigences environnementales.

82 - Ces propositions sont soumises à l'A.M.A., afin qu'un projet définitif soit élaboré après information publique, consultation des partenaires sociaux et institutionnels et des associations concernées, et audition des municipalités. 
83 - Elles sont de nouveau remises au C.A.I.E., qui les transmet au Conseil du gouvernement, par l'intermédiaire de la Commission déléguée pour les Affaires économiques, pour leur approbation définitive.

84 * Dans le décret 11/90 du 30 janvier 1990, qui fixe les modalités de création des Assemblées dirigeantes des Parcs naturels et les lignes de base des P.R.U.G., la procédure relative à ces Plans est la suivante :

- Approbation provisoire par l'Assemblée dirigeante du Parc.

86 - Soumission aux formalités d'information publique.

87 - Transmission au Conseil du gouvernement pour approbation définitive.

88 Attendu les dispositions de la loi 4/89 dans son article 19.1, les P.R.U.G. doivent être portés à la connaissance de l'administration urbaine, avant leur approbation définitive. * Le caractère global conféré au processus de planification dans les vingt Parcs naturels concernés, la mise en cohérence dans la coordination entre les différents départements administratifs à partir desquels l'action du gouvernement andalou est mise en oeuvre, ainsi que la similitude de la procédure établie pour l'approbation définitive des deux documents (P.O.R.N. et P.R.U.G.) et la possibilité que cette approbation se réalise dans le cadre d'un même décret, ont abouti à structurer les plans de manière analogue et à homogénéiser leurs effets, champ d'application, durée, etc., ainsi que la procédure évoquée plus haut, en tenant compte des particularités et caractéristiques de chaque Parc.

90 La mise en oeuvre de tout le processus a imposé une série de procédures complexes, dont les particularités les plus notables ont été les suivantes :

91 - L'élaboration des avant-projets des P.O.R.N et P.R.U.G des Parcs naturels, du fait même de leur dimension, a supposé le rassemblement et la compilation d'informations sur le milieu physique, biotique, socio-économique et sur l'habitat, et la création d'une cartographie caractérisant les différentes zones de ces espaces, dans ces $15,8 \%$ du territoire andalou. Ceci a supposé un effort important, non seulement de coordination et de recherche, mais aussi de traitement et de synthèse de l'information obtenue.

92 - Les premières propositions des P.O.R.N, une fois élaborées, ont été remises au C.A.I.E., pour son information et son approbation, ayant pour effet d'engager la procédure.

93 - L'exigence de coordonner les différents départements administratifs à partir desquels l'action du gouvernement andalou est mise en oeuvre a conduit à la création de groupes de travail, par le même décret que le C.A.I.E. En se réunissant, ces groupes doivent harmoniser les objectifs des différentes politiques sectorielles qui doivent être menées à bien dans ces espaces.

94 - De manière simultanée, un avant-projet des P.R.U.G. a été envoyé aux différentes Assemblées dirigeantes, qui en tant qu'organes participants à l'Agence de l'Environnement, devaient contribuer à l'élaboration de ces documents. Ces Assemblées regroupent des représentants de l'administration locale, provinciale, et de différentes administrations sectorielles, ainsi que des secteurs productifs et sociaux les plus représentatifs des différents espaces, dans le but de les faire participer au processus et de les informer des actions qui se dérouleraient dans les Parcs.

95 Peut-être est-ce là la séquence la plus complexe du processus, puisqu'elle suppose le consensus, qui n'est pas toujours aisé, entre des positions, points de vue et opinions émanant de secteurs et d'intérêts historiquement en conflit, comme les agriculteurs et 
les éleveurs, les syndicats et le patronat, les écologistes et les chasseurs, les municipalités de différentes tendances politiques, etc. Ce regroupement et ces divergences ont ralenti le processus, car ces Assemblées avaient également pour tâche d'informer les P.O.R.N., cette fonction étant établie dans le décret même de leur création (décret 11/90 du 30 janvier).

- Ultérieurement, et pour satisfaire aux dispositions établies légalement en matière de transmission des documents, ceux-ci ont été envoyés aux différentes Mairies dont les territoires municipaux étaient concernés par le classement en Parc naturel.

97 - Par ailleurs, l'obligation légale de communiquer les plans aux partenaires sociaux et institutionnels concernés (décret du 30 janvier, et accord du Conseil de Gouvernement de la même date) a entraîné, du fait du manque de précision de la législation sur ce point, l'établissement d'une liste des organismes et institutions impliqués, qui rassemble tous ceux qui sont susceptibles d'être concernés par le classement en Parc naturel. Leur nombre oscille entre vingt et vingt-cinq organismes pour chaque Parc, en tenant compte de ceux qui sont représentés dans les Assemblées dirigeantes: entre autres les Confederaciones hidrográficas ${ }^{8}$, la Confédération patronale, les syndicats, les associations écologistes...

En guise de conclusion

98 Quelques interprétations du processus long et complexe que nous venons de décrire peuvent être formulées en conclusion. Ayant été vérifiées sur plusieurs espaces naturels (Ojeda Rivera J. F., 1993), elles semblent valables pour l'ensemble de la région andalouse.

99 * Du fait du manque de cohésion de la société civile andalouse, le classement officiel des espaces protégés n'a pu être fondé sur un consensus préalable avec la population qui habitait les régions concernées, ou y détenait des intérêts concrets.

* La connaissance des réalités physiques, économiques et sociales des espaces régionaux concernés, fondée sur des études d'impact et des plans directeurs, a conduit le Gouvernement andalou à multiplier les inventaires et les catalogues des espaces à protéger. Ils ont été réalisés par le biais des Plans spéciaux de protection du milieu physique et du Catalogue des espaces protégés (1982-1987), ou les Plans directeurs des espaces littoraux (1985-1990), ou encore les Bases pour l'aménagement du territoire (1986-1990).

$101{ }^{*}$ L'ensemble de ces approches a conduit à la Loi 2/89 proposée par le Gouvernement régional andalou, afin que le Parlement andalou puisse «approuver l'inventaire des Espaces naturels protégés d'Andalousie et mettre en place les mesures appropriées à leur protection ». Pour être en conformité avec cette loi, le Gouvernement andalou a été tenu de développer tout un processus de planification qui ait une légitimité démocratique, destiné à combler le déficit de consensus social initial.

$102{ }^{*}$ Ce processus de planification des espaces naturels andalous traduit bien les difficultés de cohésion de la société civile andalouse. Néanmoins, il paraît constituer un bon terrain d'étude et/ou d'expérimentation pour que cette cohésion de la société civile aille s'améliorant et pour que la gestion des espaces naturels soit moins technocratique et plus consensuelle. Peut-être le cadre de référence présenté ici contribuera-t-il à une meilleure compréhension des problèmes et des difficultés. 


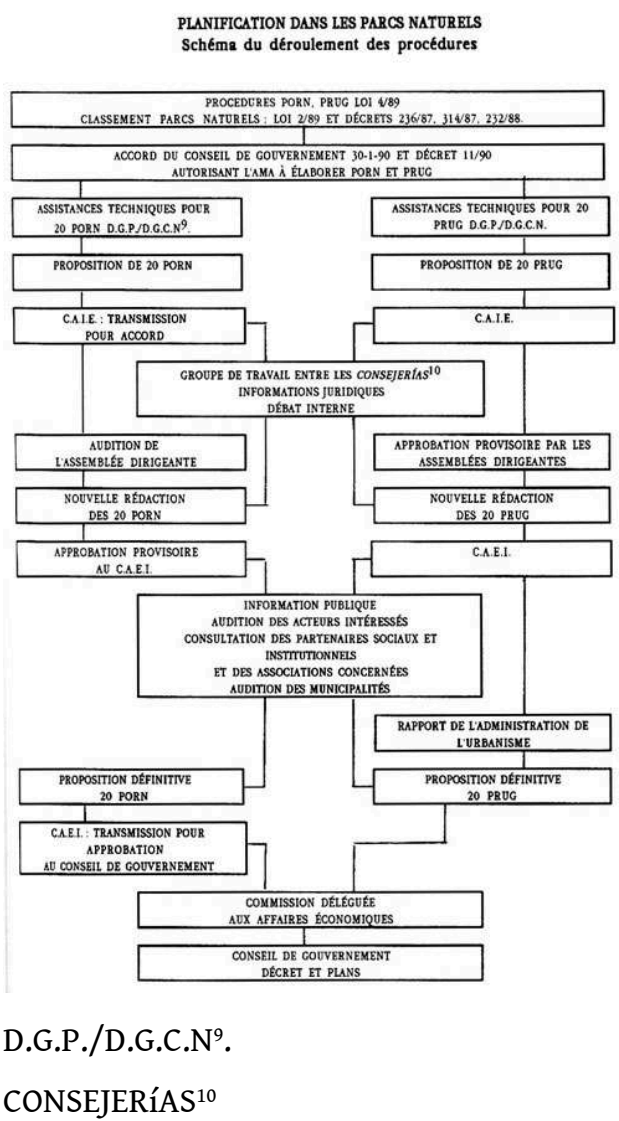

\section{BIBLIOGRAPHIE}

AMA (1990), Guía de los Espacios Naturales de Andalucía, Junta de Andalucía, Ed. Incafo.

CARRILLO, E. (1992), « Desarrollo local y Medio Ambiente », IV Congreso Nacional de Economía : Desarrollo Económico y Medio Ambiente, Séville, Ed. Aranzadi.

DELGADO CABEZA, M. \& MORILLAS RAYA, A. (1991), Metodología para la Incorporación del Medio Ambiente en la Planificación económica, Séville, Monografías de Economía y Medio Ambiente, 1 , AMA.

DIAZ OJEDA, F. (1993), « El proceso de planificación en Parques naturales : el marco andaluz », I Conferencia general sobre gestión autonómica del Medio Ambiente, Séville.

GRUPO DE INVESTIGACION : CIENCIAS TERRITORIALES Y AMBIENTALES (1992), « Políticas medioambientales y desigualdades territoriales en la CEE », , IV Congreso Nacional de Economía : Desarrollo Económico y Medio Ambiente, Séville, Ed. Aranzadi.

Loi 4/89 du 27 mars 1989, " De Conservación de los Espacios Naturales y de la Flora y Fauna Silvestres », BOE, 74, 1989. 
Loi 2/89 du 18 juillet 1989, "Por la que se aprueba el inventario de Espacios Naturales Protegidos de Andalucía y se establecen medidas adicionales para su protección », BOJA, 305, 1989.

MARTINEZ SALCEDO, F. (1992), « Gasto público e inversión privada en Medio Ambiente : Andalucía ante el mercado único europeo », IV Congreso Nacional de Economía : Desarrollo Económico y Medio Ambiente, Séville, Ed. Aranzadi.

MUÑOZ MARTINEZ, A. (1992), « Diseño e instrumentos de planificación en Espacios Naturales Protegidos », Seminario «El desarrollo sostenible : los Espacios Naturales y la gestión de los Espacios Protegidos ", Barcelone.

OJEDA RIVERA, J.F. (1992), « Sociedades y Territorios de la España Turística Tradicional. Una reflexión desde Andalucía hacia el futuro ", Revista de Estudios Regionales, 32, Universidades Andaluzas, p. 225-247.

OJEDA RIVERA, J.F. (1993), Doñana : esperando a Godot, Séville, Instituto de Desarollo Regional, Cuaderno 31.

PAREDES RUIZ, C. \& DIAZ OJEDA, F. (1992), « La intervención pública en los Espacios Naturales Protegidos de Andalucía ", IV Congreso Nacional de Economía : Desarrollo Económico y Medio Ambiente, Séville, Ed. Aranzadi.

PARRA SUPERVIA, F. (1990), « La política de Espacios Naturales : una historia ambigua », Revista Ciudad y Territorio, 83.

Résolution du 12 juin 1992, de la Agencia de Medio Ambiente, «por la que se emplaza para información pública a todos aquellos interesados en el Plan de Ordenación de los Recursos Naturales y Plan Rector de Uso y Gestión de los Parques Naturales : Sierra Nevada, Sierra Mágina, Acantilado y Pinar de Barbate, Sierra María-Los Velez, Sierras Subbéticas y Montes de Málaga », BOJA, 69, 1993.

\section{NOTES}

1. Plan de Ordenación de los Recursos Naturales.

2. Plan Rector de Uso y Gestión.

3. Plan de Desarrollo Integral.

4. Programas de Fomento.

5. Comarcas : espaces n'ayant pas d'équivalent dans le découpage territorial français, mais pouvant être rapproché à la fois de la Petite Région Agricole et du Bassin d'emploi (NDLR).

6. Instituto de Fomento de Andalucía.

7. Agencia de Medio Ambiente.

8. Assimilables à des Agences de bassins, mais qui s'en différencient surtout par leur aspect moins multi-partenarial (NDLR).

9. Dirección general de planificación et Dirección general de conservación de la naturaleza de la AMA.

10. Entité politique régionale assimilable à un « ministère » régional. 


\section{RÉSUMÉS}

Classer près du cinquième du territoire andalou au titre d'espaces naturels protégés a constitué pour l'Administration autonome une décision risquée et de très vaste envergure. Doter de tels espaces d'un ensemble de réglementations souples et opérationnelles est apparu comme un défi, de tels instruments étant considérés comme des facteurs de dynamisation et comme des moyens de lutter contre la marginalité sociale et économique des populations concernées.

\section{Preservation policies of natural spaces. The case of Andalousia.}

Almost one fifth of the andalousian territory has been classified as preserved naturel space. For the administration of the autonomous community, it was a risky decision and a broad prospect. Providing such spaces with a set of rather flexibles norms was a challenge. Such tools are considered as dynamic incentives liable to fight socio-economic marginality of the inhabitants.

\section{Politícas de espacios naturales protegidos : el marco andaluz.}

La declaración de cerca de una quinta parte del territorio andaluz como Espacio Natural Protegido ha constituido una decisión arriesgada y de enorme embergatura acometida por la Administración Autonómica. La tarea de dotar de un cuerpo normativo y de una agilidad operativa a tales espacios protegidos aparece como un reto, ya que se consideran como factores de dinamización y superación de la marginalidad social y económica en que se hayan inmersas las poblaciones afectadas.

\section{INDEX}

Mots-clés : Espace, Espagne, Espaces protégés, Andalousie, politiques de protection de l'espace, Communautés autonomes espagnoles, Procédures, Développement

Keywords : development, Spain, Andalousia, preservation policies of natural spaces, Preserved spaces, Spanish autonomous communities, Process

Palabras claves : España, Espacios protegidos, Andalucia, políticas de protección del espacio, Comunidades autónomas españolas, Trámites, Desarollo

\section{AUTEURS}

\section{FRANCISCO JOSÉ DÍAZ OJEDA}

Doctorant en géographie régionale, il est assistant technique en planification des espaces naturels protégés au Conseil de l'environnement de l'Assemblée d'Andalousie.

\section{JUAN FRANCISCO OJEDA RIVERA}

Professeur au département de géographie physique et régionale de l'Université de Séville. Ses travaux sont centrés sur l'analyse des relations entre espaces naturels et sociétés locales. 